\title{
Strongly interacting vector bosons at the CERN LHC: Quartic anomalous couplings
}

\author{
A. S. Belyaev, ${ }^{1,2}$ O. J. P. Éboli, ${ }^{1}$ M. C. Gonzalez-Garcia, ${ }^{3}$ J. K. Mizukoshi, ${ }^{4}$ S. F. Novaes, ${ }^{1}$ and I. Zacharov ${ }^{5}$ \\ ${ }^{1}$ Instituto de Física Teórica, Universidade Estadual Paulista, Rua Pamplona 145, 01405-900 São Paulo, Brazil \\ ${ }^{2}$ Skobeltsyn Institute of Nuclear Physics, Moscow State University, 119899 Moscow, Russian Federation \\ ${ }^{3}$ Instituto de Física Corpuscular IFIC/CSIC, Departament de Física Teòrica, Universitat de València, \\ 46100 Burjassot, València, Spain \\ ${ }^{4}$ Instituto de Física, Universidade de São Paulo, Caixa Postal 66318, 05315-970, São Paulo, Brazil \\ ${ }^{5}$ Silicon Graphics European Headquarters, Chemin des Avouillons 30, 1196 Gland, Switzerland
}

(Received 5 May 1998; published 11 December 1998)

\begin{abstract}
We analyze the potential of the CERN Large Hadron Collider to study anomalous quartic vector-boson interactions through the production of vector-boson pairs accompanied by jets. In the framework of $\mathrm{SU}(2)_{L} \otimes \mathrm{U}(1)_{Y}$ chiral Lagrangians, we examine all effective operators of order $p^{4}$ that lead to new fourgauge-boson interactions but do not alter trilinear vertices. In our analyses, we perform the full tree-level calculation of the processes leading to two jets plus vector-boson pairs, $W^{+} W^{-}, W^{ \pm} W^{ \pm}, W^{ \pm} Z$, or $Z Z$, taking properly into account the interference between the standard model and the anomalous contributions. We obtain the bounds that can be placed on the anomalous quartic interactions and we study the strategies to distinguish the possible new couplings. [S0556-2821(99)06201-3]

PACS number(s): $12.60 . \mathrm{Cn}$
\end{abstract}

\section{INTRODUCTION}

The standard model (SM) of electroweak interactions, based on the $\mathrm{SU}(2)_{L} \otimes \mathrm{U}(1)_{Y}$ gauge symmetry, has accomplished an impressive agreement between its predictions for the fermion-vector-boson couplings and all the recent experimental data [1]. Notwithstanding, the tests of the triple and quartic bosonic interactions still lack the same accuracy to further confirm the local gauge invariance of the theory or to indicate the existence of new physics beyond the SM.

The interactions responsible for electroweak symmetry breaking play an important role in gauge-boson scattering at high energies because they are an essential ingredient to avoid unitarity violation in the scattering amplitudes of massive vector bosons at the $\mathrm{TeV}$ scale [2]. There are two possible forms of electroweak symmetry breaking which lead to different solutions to the unitarity problem: (a) there is a scalar particle lighter than $1 \mathrm{TeV}$, the standard model Higgs boson, or (b) such a particle is absent and the longitudinal components of the $W$ and $Z$ bosons become strongly interacting at high energies. In the latter case, symmetry breaking occurs due to the nonzero vacuum expectation value of some composite operators which are related with new underlying physics.

In this work we analyze the potential of the CERN Large Hadron Collider (LHC) to study deviations of the quartic vector-boson couplings from the SM predictions, assuming a strongly interacting electroweak symmetry breaking sector (SEWS). In fact, the LHC will be the first collider capable of directly studying these couplings through the scattering of gauge bosons in reactions like $p p \rightarrow q q V V \rightarrow V V j j$ [3-5], with $V=W^{ \pm}$or $Z^{0}$. Studies of quartic couplings will also be possible at future $e^{+} e^{-}$colliders [6-10], and also in $e \gamma$ [11] and $\gamma \gamma$ collisions [12]. Notwithstanding, at present, this sector of the SM can only be indirectly bounded by the precise measurements of the electroweak parameters $[13,14]$.

In this paper we assume that there are no new light reso- nances at the LHC energy scale, which means that the $\mathrm{SU}(2)_{L} \otimes \mathrm{U}(1)_{Y}$ gauge symmetry is nonlinearly realized. In this case, the electroweak sector must be parametrized in terms of electroweak chiral Lagrangians. We study the complete set of dimension-four operators contributing only to quartic vector-boson couplings and we estimate the sensitivity of the LHC to search for deviations from the SM predictions.

We present the results for the full tree-level calculation of the processes $p p \rightarrow V V+2$ jets, with $V=W^{ \pm}, Z^{0}$, taking properly into account the interference between the SM and anomalous quartic contributions. This improves the previous studies of SEWS at the LHC [3-5] which relied upon the equivalence theorem [15] or/and the effective $W$-boson approximation [16]. Moreover, we performed our calculation both in the unitary and 't Hooft-Feynman gauges, and we also included the efficiencies for detecting the leptons originating from the vector boson decays.

In our analyses we obtain the allowed range of the coefficient of each anomalous quartic operator and compare the results with those coming from indirect measurements $[13,14]$, as well as the attainable limits at future $e^{+} e^{-}$colliders [6-10]. In addition to the discovery of an anomalous behavior of the cross section for the production of a vector boson pair, it is important to identify the possible source of this deviation. Depending on the particular operator(s) responsible for the deviations, we could have some hint about the underlying physics that generates this departure from the SM predictions. This can be achieved by the comparative analysis of the different reactions since distinct operators contribute differently to each possible two boson final states.

The paper is organized as follows. In the next section, we summarize the model-independent formalism and present the respective chiral Lagrangians describing the anomalous quartic couplings among the gauge bosons. In Sec. III, we analyze both the signals and backgrounds involved in the production of a vector boson pairs accompanied by two jets. 
We also establish the best cuts to improve the signal over background ratio. Our final results for the cross sections are presented in Sec. IV, in terms of the chiral Lagrangian coefficients. The final section contains our general conclusions.

\section{CHIRAL LAGRANGIANS}

When the Higgs boson is a strongly interacting particle or when it is absent from the physical particle spectrum, one is led to consider the most general effective Lagrangian which employs a nonlinear representation of the spontaneously broken $\mathrm{SU}(2)_{L} \otimes \mathrm{U}(1)_{Y}$ gauge symmetry [17]. The resulting chiral Lagrangian is a nonrenormalizable nonlinear $\sigma$ model coupled in a gauge-invariant way to the Yang-Mills theory. This model-independent approach incorporates by construction the low-energy theorems [18], which predict the general behavior of Goldstone boson amplitudes, irrespective of the details of the symmetry-breaking mechanism. This lowenergy effective theory should be valid up to some energy scale smaller than $4 \pi v \simeq 3 \mathrm{TeV}$, where new physics would come into play to avoid unitarity violation in vector-boson scattering [2].

In order to specify the effective Lagrangian, one must fix the symmetry-breaking pattern. We considered that the system presents a global $\mathrm{SU}(2)_{L} \otimes \mathrm{SU}(2)_{R}$ symmetry that is broken to $\mathrm{SU}(2)$. With this choice, following the notation of Ref.citeAppelquist, the building block of the chiral Lagrangian is the dimensionless unimodular matrix field $\Sigma(x)$, which transforms under $\mathrm{SU}(2)_{L} \otimes \mathrm{SU}(2)_{R}$ as $(2,2)$,

$$
\Sigma(x)=\exp \left[i \frac{\varphi^{a}(x) \tau^{a}}{v}\right],
$$

where the $\varphi^{a}$ fields are the would-be Goldstone fields and $\tau^{a}(a=1,2,3)$ are the Pauli matrices. The $\mathrm{SU}(2)_{L} \otimes \mathrm{U}(1)_{Y}$ covariant derivative of $\Sigma$ is defined as

$$
D_{\mu} \Sigma \equiv \partial_{\mu} \Sigma+i g \frac{\tau^{a}}{2} W_{\mu}^{a} \Sigma-i g^{\prime} \Sigma \frac{\tau^{3}}{2} B_{\mu}
$$

The lowest-order terms in the derivative expansion of the effective Lagrangian are

$$
\mathcal{L}^{(2)}=\frac{v^{2}}{4} \operatorname{Tr}\left[\left(D_{\mu} \Sigma\right)^{\dagger}\left(D^{\mu} \Sigma\right)\right]+\beta_{1} g^{\prime 2} \frac{v^{2}}{4}\left(\operatorname{Tr}\left[T V_{\mu}\right]\right)^{2},
$$

where we have introduced the auxiliary quantities $T$ $\equiv \Sigma \tau^{3} \Sigma^{\dagger}$ and $V_{\mu} \equiv\left(D_{\mu} \Sigma\right) \Sigma^{\dagger}$ which are $\mathrm{SU}(2)_{L}$ covariant and $\mathrm{U}(1)_{Y}$ invariant. Notice that $T$ is not invariant under $\mathrm{SU}(2)_{C}$ custodial due to the presence of $\tau^{3}$.

The first term of the above equation is responsible for giving mass to the gauge bosons $W^{ \pm}$and $Z$ for $v$ $=\left(\sqrt{2} G_{F}\right)^{-1}$. The second term violates the custodial $\mathrm{SU}(2)_{C}$ symmetry and contributes to $\Delta \rho$ at the tree level, being strongly constrained by the low-energy data. This term can be understood as the low-energy remnant of the highenergy custodial symmetry-breaking physics, which has been integrated out above a certain scale $\Lambda$. Moreover, at the oneloop order, it is also required in order to cancel the diver- gences in $\Delta \rho$, arising from diagrams containing a hypercharge boson in the loop [17]. This subtraction renders a finite $\Delta \rho$, although dependent on the renormalization scale.

At the next order in the derivative expansion $\left(p^{4}\right)$, there are many operators that can be written down [17]. We shall restrict our analyses to the ones that exhibit genuine quartic vector-boson interactions, i.e., that do not have triple gaugeboson vertices associated to these quartic couplings. These operators are

$$
\begin{aligned}
& \mathcal{L}_{4}^{(4)}=\alpha_{4}\left[\operatorname{Tr}\left(V_{\mu} V_{\nu}\right)\right]^{2}, \\
& \mathcal{L}_{5}^{(4)}=\alpha_{5}\left[\operatorname{Tr}\left(V_{\mu} V^{\mu}\right)\right]^{2}, \\
& \mathcal{L}_{6}^{(4)}=\alpha_{6} \operatorname{Tr}\left(V_{\mu} V_{\nu}\right) \operatorname{Tr}\left(T V^{\mu}\right) \operatorname{Tr}\left(T V^{\nu}\right), \\
& \mathcal{L}_{7}^{(4)}=\alpha_{7} \operatorname{Tr}\left(V_{\mu} V^{\mu}\right)\left[\operatorname{Tr}\left(T V^{\nu}\right)\right]^{2}, \\
& \mathcal{L}_{10}^{(4)}=\frac{\alpha_{10}}{2}\left[\operatorname{Tr}\left(T V_{\mu}\right) \operatorname{Tr}\left(T V_{\nu}\right)\right]^{2} .
\end{aligned}
$$

These Lagrangian densities lead to quartic vertices involving gauge bosons and/or Goldstone bosons. In the unitary gauge, there are new anomalous contributions to the $Z Z Z Z$ vertex coming from all five operators, to the $W^{+} W^{-} Z Z$ vertex from all operators except $\mathcal{L}_{10}^{(4)}$, and to $W^{+} W^{-} W^{+} W^{-}$interaction arising from $\mathcal{L}_{4}^{(4)}$ and $\mathcal{L}_{5}^{(4)}$. Moreover, the interaction Lagrangians $\mathcal{L}_{6}^{(4)}, \mathcal{L}_{7}^{(4)}$, and $\mathcal{L}_{10}^{(4)}$ violate the $\mathrm{SU}(2)_{C}$ custodial symmetry. Notice that the quartic couplings involving photons remain untouched by the genuinely quartic anomalous interactions at the order $p^{4}$. The Feynman rules for the quartic couplings generated by these operators can be found in the last article of Ref. [17].

\section{SIGNALS AND BACKGROUNDS}

In our analyses, we studied the strongly interacting electroweak breaking sector at the LHC via the scattering of weak vector bosons that are radiated off quarks. We considered the following processes involving the four-gauge-boson interactions (4)-(8):

$$
\begin{aligned}
& p p \rightarrow W^{+} W^{-} j j, \\
& p p \rightarrow W^{-} W^{-} j j, \\
& p p \rightarrow W^{+} W^{+} j j, \\
& p p \rightarrow W^{+} Z j j, \\
& p p \rightarrow W^{-} Z j j, \\
& p p \rightarrow Z Z j j .
\end{aligned}
$$

We evaluated the complete set of QCD and electroweak scattering amplitudes for the above processes, i.e., we did not use neither the effective $W$ approximation [16] nor the equivalence theorem [15]. Therefore, we were able to keep track of the full correlation in the matrix elements, as well as the interference between the anomalous and SM contribu- 
TABLE I. The processes affected by the different quartic couplings (4)-(8).

\begin{tabular}{lccc}
\hline \hline Coupling & $W^{ \pm} W^{ \pm}$ & $W^{ \pm} Z$ & $Z Z$ \\
\hline$\alpha_{4,5}$ & Yes & Yes & Yes \\
$\alpha_{6,7}$ & No & Yes & Yes \\
$\alpha_{10}$ & No & No & Yes \\
\hline \hline
\end{tabular}

tions. Moreover, we took into account not only the electroweak contributions but also the $\mathcal{O}\left(\alpha^{2} \alpha_{S}^{2}\right)$ ones. For the sake of clarity, we show in Table I the anomalous interactions that contribute to each of the reactions (9). This table indicates the strategy that should be followed to understand the origin of the possible deviations from the SM.

The calculation of the matrix elements was performed numerically using two distinct tools. On one hand, we evaluated the scattering amplitudes in the unitary gauge using the HELAS package [19], with the SM contribution being generated by MADGRAPH [20]. In this case, we wrote special subroutines to evaluate the anomalous contributions (4)-(8) to the vector-boson self-interactions. On the other hand, the same processes were evaluated using the COMPHEP package [21]. The $p^{4}$ chiral effective Lagrangian was implemented into COMPHEP in the unitary and the 't Hooft-Feynman gauges. Despite the Feynman rules in the 't Hooft-Feynman gauge being cumbersome, this gauge maximizes the COMPHEP performance and allows us to double check our calculations by comparing the results in two gauges. The results from HELAS/MADGRAPH and COMPHEP were confronted and they indeed agreed.

The evaluation of the processes (9) requires a very large computing power. The complexity of this calculation can inferred from the large number of diagrams involved. For instance, there are 1918 Feynman diagrams contributing to the $W^{+} W^{-}$final state, while for $W^{+} Z$ there are 1503 , and 978 for $Z Z$. As an illustration, we present, in Fig. 1, the complete set of Feynman diagrams for the subprocess $u u$ $\rightarrow W^{+} W^{+} d d$ which contributes to the $W^{+} W^{+}$production (9c). The first diagram in this figure receives contributions from the anomalous interactions, giving rise to the signal, while all other graphs correspond to QCD and electroweak backgrounds. We neglected in our analyses the small contribution coming from subprocesses exhibiting two sea quarks in the initial state.

Strongly interacting symmetry breaking sectors modify the dynamics of longitudinal vector bosons. However, it is impossible to determine the polarization of vector bosons on an event-by-event basis, and consequently, we have to work harder to extract the SEWS signal. Taking into account that the electroweak production of transversely polarized vector bosons is approximately independent of the Higgs boson
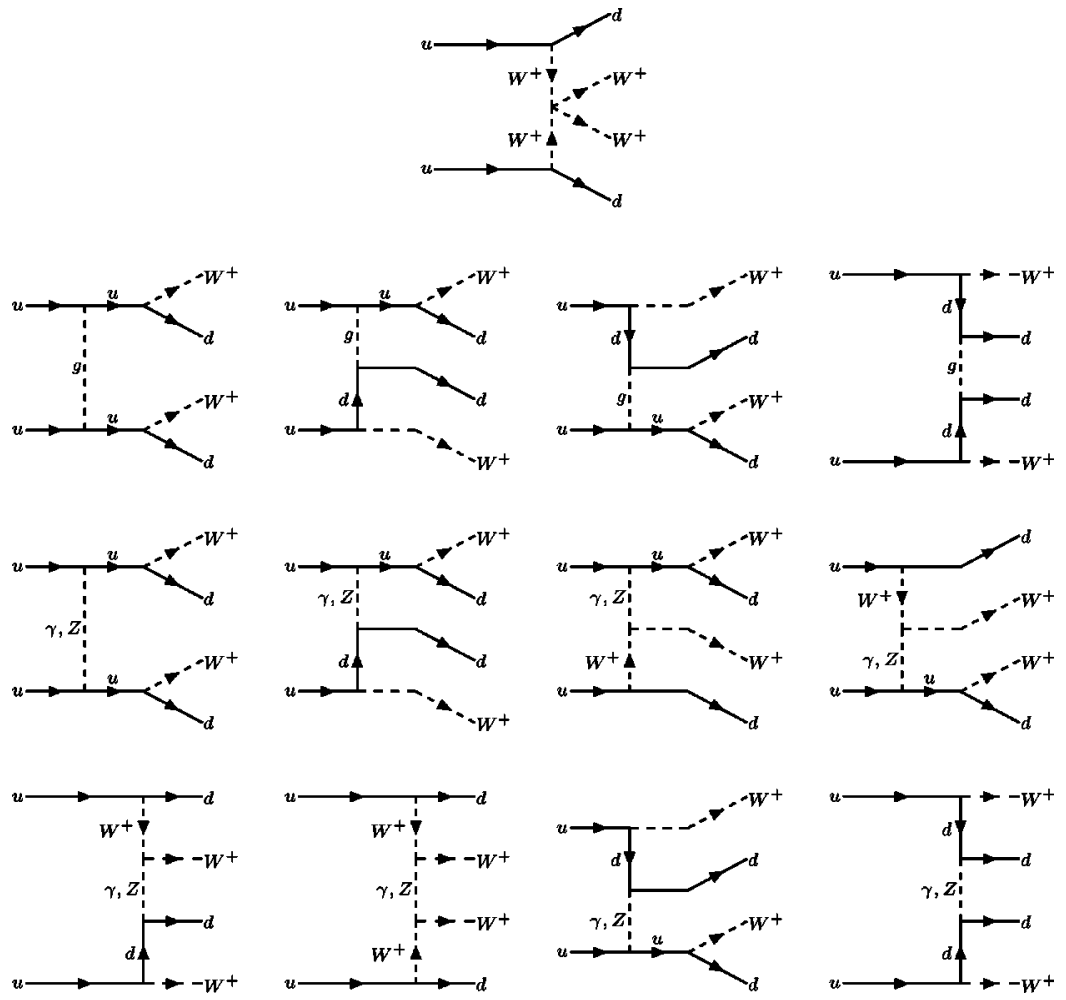
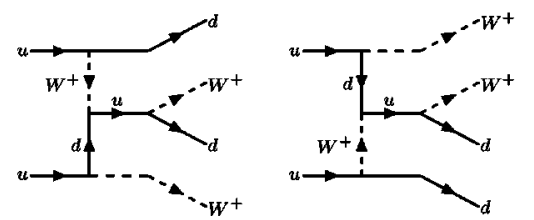

FIG. 1. Complete set of Feynman diagrams contributing to the process $u u \rightarrow W^{+} W^{+} d d$. 
mass, and that the $V_{L} V_{L}$ production is small for light Higgs bosons [4], we define the signal for SEWS as an excess of events in the $V V$ scattering channels with respect to the SM model with a light Higgs boson:, i.e.,

$$
\sigma_{\text {signal }} \equiv \sigma\left(\alpha_{i}\right)-\left.\sigma_{S M}\right|_{M_{H}=100 \mathrm{GeV}},
$$

where we sum over the vector-boson polarizations. In principle, we might have a signal even for $\alpha_{i} \equiv 0$ since there is no Higgs boson in our model to cut off the growth of the scattering amplitudes. In this case, we should also study whether it is possible to establish that the anomalous couplings $\alpha_{i}$ are compatible with zero or not.

In the effective- $W$ approximation [16], the signal is described by the scattering $V_{L} V_{L} \rightarrow V_{L} V_{L}$. This process, however, does not respect the unitarity of the partial-wave amplitudes $\left(a_{\ell}^{I}\right)$ at large subprocess center-of-mass energies $M_{V V}[2,15]$. Therefore, the chiral expansion is valid only for values of $M_{V V}$ and $\alpha_{i}$ such that $\left|a_{\gamma}^{I}\right| \leq 1 / 2$. For higher $V V$ invariant masses, rescattering effects are important to unitarize the amplitudes. Taking into account this fact, we conservatively restricted our analyses to invariant masses $M_{V V}$ $<1.25 \mathrm{TeV}$. In the cases where it is not possible to reconstruct the $V V$ invariant mass from the leptonic decay products, this requirement corresponds to a sharp-cutoff unitarization [22].

Since we evaluated the full matrix elements for the processes (9), summed over the vector-boson polarizations, several backgrounds were automatically included, e.g., the $\mathcal{O}\left(\alpha^{4}\right)$ and $\mathcal{O}\left(\alpha^{2} \alpha_{S}^{2}\right) \quad$ irreducible backgrounds $q q$ $\rightarrow q q V_{T} V_{T}\left(V_{L} V_{T}\right)$. Another important background is topquark pair production, i.e., $q q(g g) \rightarrow t \bar{t} \rightarrow W^{+} W^{-} b \bar{b}$ which was also taken into account, since we considered the $W^{+} W^{-} b \bar{b}$ final state. Moreover, triple gauge boson production also contribute to the $V V j j$ signature when one of the three boson decays hadronically. In addition to that we also evaluated the "continuum" $V V$ production, $q q(g g)$ $\rightarrow g g V V$, where the vector bosons are produced in association with gluons. In principle, we should explicitly include further backgrounds like the associated production of $t \bar{t}$ pairs accompanied by a $W^{ \pm}$or a $Z$, however, these contributions are negligible once we applied the jet veto and tag cuts described below [4].

One should stress the importance of the jet-tagging and jet-vetoing cuts since the background can be efficiently suppressed by cutting in the jet rapidities and momenta [4]. In order to understand that, we must recall that the spectra of transversely $\left(f_{W / e}^{T}\right)$ and longitudinally $\left(f_{W / e}^{L}\right)$ polarized $W$ in the effective $W$ approximation are given by

$$
f_{W / e}^{T}\left(x, p_{T}\right)=\frac{\alpha}{4 \pi \sin ^{2} \theta_{W}} \frac{1+(1-x)^{2}}{2 x} \frac{p_{T}^{2}}{\left[p_{T}^{2}+(1-x) M_{W}^{2}\right]^{2}},
$$

$$
f_{W / e}^{L}\left(x, p_{T}\right)=\frac{\alpha}{4 \pi \sin ^{2} \theta_{W}} \frac{1-x}{x} \frac{(1-x) M_{W}^{2}}{\left[p_{T}^{2}+(1-x) M_{W}^{2}\right]^{2}},
$$

where $p_{T}$ is the transverse momentum of the $W^{ \pm}$(jet). From the above expressions, we can learn that the transversely polarized $W$ 's possess a higher $p_{T}$ than the longitudinally polarized ones. Moreover, the spectator jets associated with $W_{L}^{ \pm}$are produced at large rapidities since their energies are of the order of $\mathrm{TeV}$.

Forward (backward) jets are characteristic configurations of the signal. At the same time, jets coming from the signal are well separated and their $p_{T}$ distribution does not peak near zero because of massive vector-boson propagators. On the other hand, the situation is the opposite for some backgrounds: either their $p_{T}$ distributions peak at small values due to photon, gluon or light quark $t$-channel exchange, or they have the tendency to be close to each other since the jets originate from gluon or photon splitting. This remarkable difference between the signal and the backgrounds allows us to substantially reduce the latter by requiring the tagging of forward jets. We can further reduce backgrounds, like $t \bar{t}$ and $V V V$, by vetoing large jet activity in the central region of the detector [23].

In Fig. 2, we show some kinematical distributions for the process $p p \rightarrow W^{+} Z j j$ (9d). Figure 2(a) contains the pseudorapidity distribution of the jets, while we exhibit the $p_{T}$ (energy) distribution of the jets in Figs. 2(b),2(c), and the invariant mass distribution of $W^{+} Z$ pairs in Fig. 2(d). From these figures we can see that the jets associated with the signal are produced at large rapidities and carry a larger amount of energy, illustrating the importance jet-tagging and jetvetoing cuts.

In order to suppress the backgrounds and enhance the signal for anomalous quartic interactions we studied several kinematical distributions for the processes (9), applying different cuts on the final-state particles. Our results indicate that the cuts presented in Ref. [4] are able to improve considerably the signal/background ratio. We applied the following set of kinematical cuts, keeping those from the above-mentioned paper and also suggesting some additional ones that could allow further suppression of the backgrounds.

(i) We required the existence of two jets satisfying $p_{T}$ $>20 \mathrm{GeV},|\eta|<5$, and separated by $\Delta R \equiv \sqrt{\left(\Delta \eta^{2}+\Delta \phi^{2}\right)}$ $>0.5$. The cut in $p_{T}$ is important not only to guarantee that the jets will be well defined, but also to suppress the background due to the photon and gluon exchanges in the $t$ channel. At the same time, the $\Delta R$ cut is necessary, combined with the $p_{T}$ one, to remove the singularity coming from gluon splitting in some background subprocesses.

(ii) We applied the jet-tagging and jet-vetoing cuts suggested by Bagger et al. [4], i.e.,

$$
\begin{gathered}
E\left(j_{\text {tag }}\right)>0.8 \mathrm{TeV} \quad\left(\text { except for } W^{ \pm} W^{ \pm}\right), \\
3.0<\left|y\left(j_{\text {tag }}\right)\right|<5.0, \quad p_{T}\left(j_{\text {tag }}\right)>40 \mathrm{GeV}, \\
p_{T}\left(j_{\text {veto }}\right)>60 \mathrm{GeV} \quad\left(30 \mathrm{GeV} \text { for } W^{+} W^{-}\right), \\
\left|y\left(j_{\text {veto }}\right)\right|<3.0 .
\end{gathered}
$$



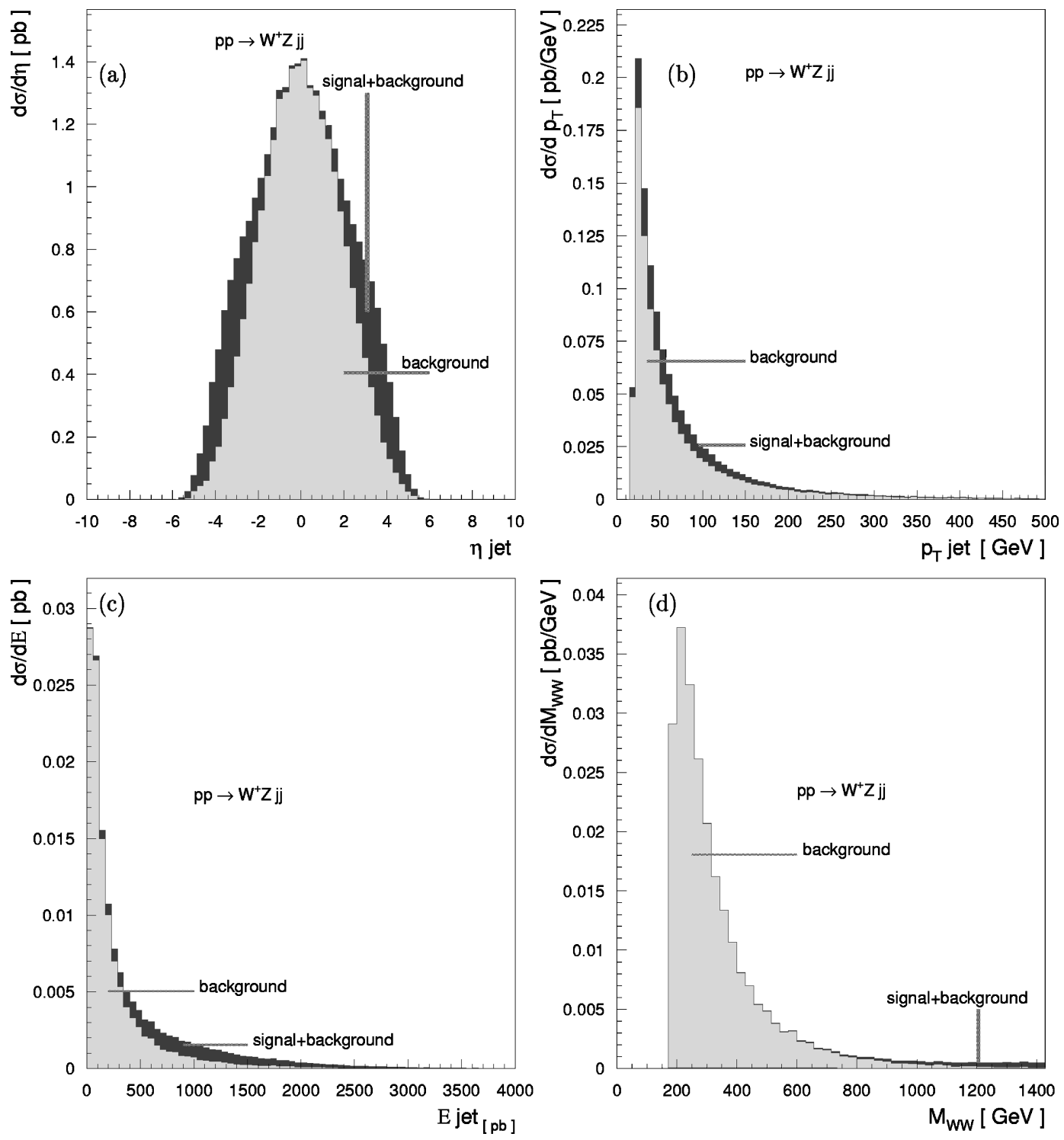

FIG. 2. Kinematical distributions for the process $p p \rightarrow W^{+} Z j j$ : (a) pseudorapidity of the jets $\left(\eta_{j}\right)$; (b) transverse momentum of the jets $\left(p_{T_{j}}\right)$; (c) energy of the jets $\left(E_{j}\right)$; and $(\mathrm{d})$ invariant mass of the $W^{+} Z$ pair $\left(M_{W W}\right)$. The light gray area stands for the background while the dark area represents the background plus the signal associated to $\alpha_{4}=0.03$. We required that $p_{T}^{\text {jet }}>20 \mathrm{GeV}$ and the jet separation $\Delta R_{j j}$ $>0.5$.

$t \bar{t}$ production gives rise to a quite large background to the $W^{+} W^{-} j j$ signal, and consequently, the requirement of a more stringent $p_{T}\left(j_{\text {veto }}\right)$ cut for this process is important to improve the signal/background ratio.

(iii) $\mathrm{We}$ also required the invariant mass of the vector boson pair to be in the range $0.5<M_{V V}<1.25 \mathrm{TeV}$. The upper limit of this cut is quite important since it prevents the effective operators (4)-(8) to be used in a energy regime where unitarity is violated and rescattering effects become important. The lower limit of this cut aims to reduce the background (see Fig. 2).

In this work we considered the "gold-plated" events where the $W$ 's and $Z$ 's decay into electrons or muons, ignoring final states associated with the hadronic decay of the vector bosons. In order to make a more realistic estimation of the limits that can be imposed on the anomalous parameters, one should take into account the detection efficiency of the final-state leptons. This problem was studied in Ref. [24] for $W^{ \pm}$and $Z$ decays in Higgs production processes. Imposing that the leptons satisfy the following cuts:

$$
\left|\eta^{\prime}\right|<2, \quad p_{T}^{\prime}>100 \mathrm{GeV}, \quad \text { and } \quad p_{T}^{\text {miss }}>100 \mathrm{GeV}
$$

the detection efficiency for leptons originating from $W(Z)$ decays is $43 \%(52 \%)$ [24]. We also took into account the branching ratios of $W^{ \pm}$and $Z$ into electrons or muons ( $\ell$ $=e$ or $\mu)$,

$$
\begin{aligned}
& \mathrm{BR}\left(W W \rightarrow \ell \bar{\nu}_{\ell} \bar{\ell}_{\ell}\right)=4.7 \%, \\
& \mathrm{BR}\left(W^{+} Z \rightarrow \ell \bar{\nu}_{\ell} \ell \bar{\ell}\right)=1.5 \%,
\end{aligned}
$$




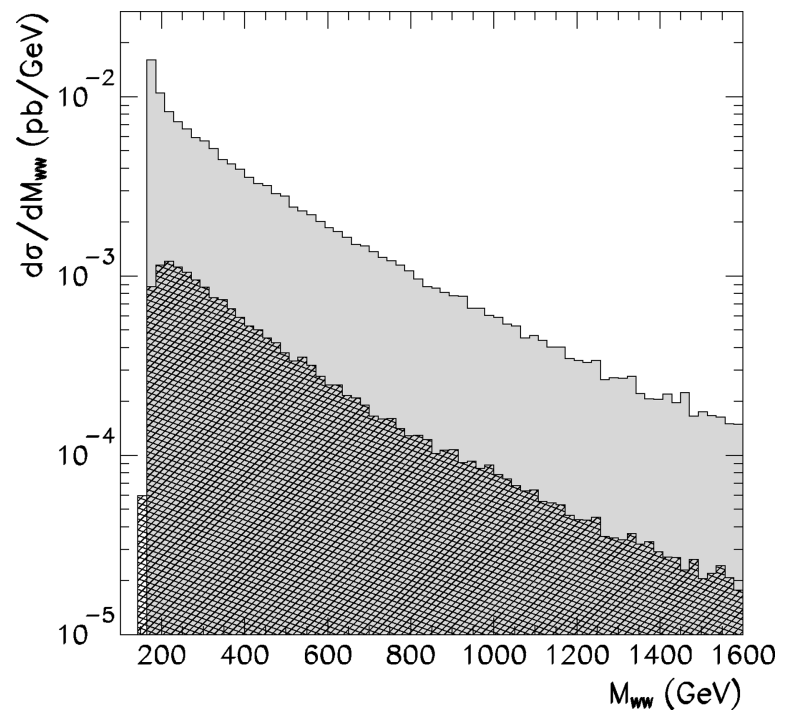

FIG. 3. $M_{W W}$ invariant mass distribution for the process $p p$ $\rightarrow W^{+} W^{+} j j$ in the effective $W$ approximation (light grey histogram) and in the full calculation (dark grey histogram) with $p_{T}^{W}$ $>20 \mathrm{GeV}$ cut.

$$
\mathrm{BR}(Z Z \rightarrow 4 \ell)=0.45 \%
$$

At this point it is important to address the difference between the results obtained using the full matrix elements and the effective $W$ approximation. First, as it is well known, the effective $W$ approximation overestimates the total yield of $W$ pairs in the production of a heavy Higgs boson [25]. In order to verify that this is still true when there is no resonances, we computed the process $p p \rightarrow W^{+} W^{+} j j$ in the standard model, both in the effective $W$ approximation and with the full matrix element, imposing only the cut $p_{T}^{W}>20 \mathrm{GeV}$ to avoid divergences in the effective $W$ calculation. We obtain

$$
\begin{aligned}
\sigma_{\text {full }}\left(p p \rightarrow W^{+} W^{+} j j\right) & =0.385 \mathrm{pb}, \\
\sigma_{\text {eff } \mathrm{W}}\left(p p \rightarrow W^{+} W^{+} j j\right) & =2.92 \mathrm{pb},
\end{aligned}
$$

which shows that the result in the effective $W$ approximation is almost one order of magnitude larger than the full calculation. This discrepancy reduces when the $p_{T}^{W}$ cut is increased or when the $W^{\prime}$ 's are required to be central [25]. Nonetheless, we should remember that we do not impose any cut on the $W$ rapidity or $p_{T}$ in our realistic complete calculations.

In Fig. 3, we compare the $W W$ invariant mass distribution obtained using the effective $W$ approximation and performing the full calculation, imposing only the $p_{T}^{W}$ cut. As seen in the figure, the excess is relatively larger at small $M_{W W}$. In our analyses of the anomalous couplings, we required that $M_{V V}>500 \mathrm{GeV}$, keeping only the large $V V$ invariant masses. Notwithstanding, this cut reduces only slightly the difference between the effective $W$ approximation and the full calculation, to a factor $\sim 7$. Moreover, after this $M_{V V}$ cut, the effective $W$ approximation reproduces the $M_{V V}$ distribution shape.
TABLE II. Coefficients of the different combinations of coupling constants contributing to the total cross section in $\mathrm{pb}$ [see Eq. (13)], and also for the SM with a light Higgs $\left(M_{H}=100 \mathrm{GeV}\right)$. These results were obtained applying the cuts (i)-(iii).

\begin{tabular}{lcccccc}
\hline \hline & $W^{+} W^{-}$ & $W^{+} W^{+}$ & $W^{-} W^{-}$ & $W^{+} Z$ & $W^{-} Z$ & $Z Z$ \\
\hline$C_{\text {SM }}$ & 0.049 & 0.0044 & 0.0009 & 0.018 & 0.0070 & 0.0044 \\
$C_{0}$ & 0.050 & 0.0061 & 0.0011 & 0.019 & 0.0074 & 0.0056 \\
$C_{4}$ & 0.21 & -0.38 & -0.062 & -0.14 & -0.062 & 0.066 \\
$C_{5}$ & 0.27 & -0.19 & -0.034 & -0.12 & -0.057 & 0.20 \\
$C_{6}$ & 0.036 & & & -0.14 & -0.062 & 0.066 \\
$C_{7}$ & 0.11 & & & -0.12 & -0.057 & 0.20 \\
$C_{10}$ & & & & & & -0.00012 \\
$C_{4-4}$ & 18 & 27 & 4.3 & 14 & 5.4 & 13 \\
$C_{5-5}$ & 36 & 7.2 & 1.2 & 6.3 & 2.4 & 23 \\
$C_{6-6}$ & 0.67 & & & 14 & 5.4 & 49 \\
$C_{7-7}$ & 5.7 & & & 6.3 & 2.4 & 58 \\
$C_{10-10}$ & & & & & & 47 \\
$C_{4-5}$ & 46 & 28 & 4.4 & 11 & 4.2 & 31 \\
$C_{4-6}$ & 1.4 & & & 29 & 11 & 50 \\
$C_{4-7}$ & 3.6 & & & 11 & 4.2 & 55 \\
$C_{4-10}$ & & & & & & 47 \\
$C_{5-6}$ & 4.0 & & & 11 & 4.2 & 54 \\
$C_{5-7}$ & 12 & & & 13 & 4.8 & 69 \\
$C_{5-10}$ & & & & & & 47 \\
$C_{6-7}$ & 3.7 & & & 11 & 4.2 & 102 \\
$C_{6-10}$ & & & & & & 94 \\
$C_{7-10}$ & & & & & & 94 \\
\hline \hline
\end{tabular}

It is also important to notice that we imposed strong veto and tag cuts on the final jets in our analyses to reduce the backgrounds. However, these cuts are impossible to implement in the effective $W$ approximation, and in consequence, the only way to obtain the final number of events in this approximation is to estimate the veto and tag efficiency from some related "full'" calculation (for instance, from the full SM part as done by Barger et al.) and assume that this efficiency is the same for the anomalous contributions. This introduces some unavoidable uncertainty in the approximate calculation.

\section{RESULTS}

The most general expression for the total cross sections of the processes (9) can be written as

$$
\sigma=C_{0}+\sum_{j} \alpha_{j} C_{j}+\sum_{j \leqslant k} \alpha_{j} \alpha_{k} C_{j-k}
$$

where $j, k=4,5,6,7$, or 10 and $C_{0}$ is the cross section for $\alpha_{j} \equiv 0$. In our calculations, we applied the cuts (i)-(iii) and used the CTEQ3M parton distributions [26], with $Q^{2}$ equal to the invariant mass of the parton system. We present in Table II our results for the coefficients $C_{0}, C_{j}, C_{j-k}$, as well as for the SM with a Higgs boson of mass $M_{H}$ $=100 \mathrm{GeV}\left(C_{\mathrm{SM}}\right)$. 


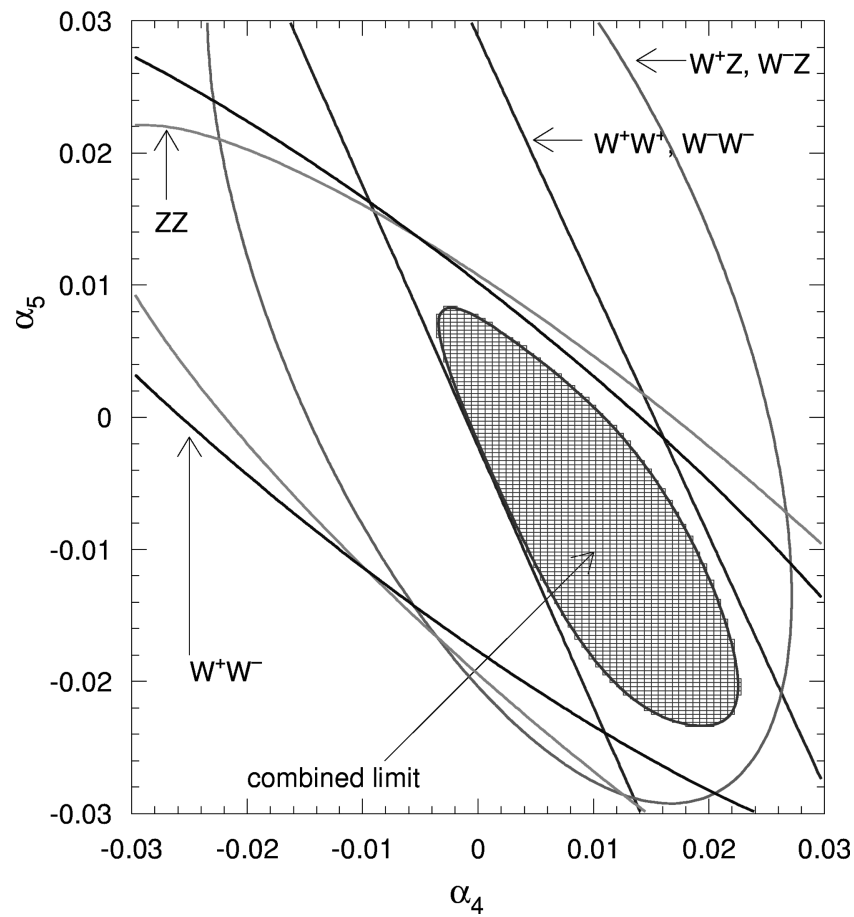

FIG. 4. 1- $\sigma$ exclusion region in the $\alpha_{4} \times \alpha_{5}$ plane for the $W^{+} W^{-}, W^{ \pm} W^{ \pm}, W^{ \pm} Z$, and $Z Z$ channels. We applied all cuts and efficiencies discussed in the text and assumed that all $\mathrm{SU}(2)_{C}$ violating couplings vanish and an integrated luminosity of $100 \mathrm{fb}^{-1}$.

Given our definition of the signal (10) and the above parametrization of the anomalous cross section we can easily obtain the LHC attainable limits on any combination of genuinely quartic anomalous couplings. We exhibit in Fig. 4 the 1- $\sigma$ exclusion region in the plane $\alpha_{4} \times \alpha_{5}$ for each process (9) independently, assuming an integrated luminosity $\mathcal{L}=100 \mathrm{fb}^{-1}$ and taking properly into account the detection efficiencies and leptonic branching ratios. In this analysis, we assumed that the $\mathrm{SU}(2)_{C}$ violating interactions vanish. As we can see, the $W^{ \pm} Z, Z Z$, and $W^{+} W^{-}$productions lead to similar bounds while the $W^{ \pm} W^{ \pm}$give rise to somewhat weaker limits. Combining all channels allow us to improve the limits by a factor of approximately 2 .

Figure 5 contains the $1-\sigma$ exclusion region in the $\alpha_{6} \times \alpha_{7}$ plane for $\alpha_{4}=\alpha_{5}=\alpha_{10}=0$ and an integrated luminosity of $100 \mathrm{fb}^{-1}$. The $W^{ \pm} W^{ \pm}$production does not give rise to any bound since these interactions possess only $Z Z Z Z$ and $W^{+} W^{-} Z Z$ anomalous couplings. Moreover, the production of $W^{+} W^{-}$pairs leads to weak bounds since these couplings contribute to this final state only through the subprocess $Z Z \rightarrow W^{+} W^{-}$, which is suppressed. The best limits come from the $Z Z$ pair production and the combined limits of $Z Z$ and $W^{ \pm} Z$ productions are only slightly better than the $Z Z$ bounds.

The anomalous interaction $\alpha_{10}$ modifies only the $Z Z$ production since it alters solely the vertex $Z Z Z Z$. We present in Fig. 6 the 1- $\sigma$ limits that can be obtained on this coupling from the $Z Z$ pair production for $\alpha_{4}=\alpha_{5}=\alpha_{6}=\alpha_{7}=0$ and an integrated luminosity of $100 \mathrm{fb}^{-1}$. Therefore, this coupling is the one that will be less constrained at the LHC.

Table III shows the limits on each coupling $\alpha_{i}, i=4,5,6$,

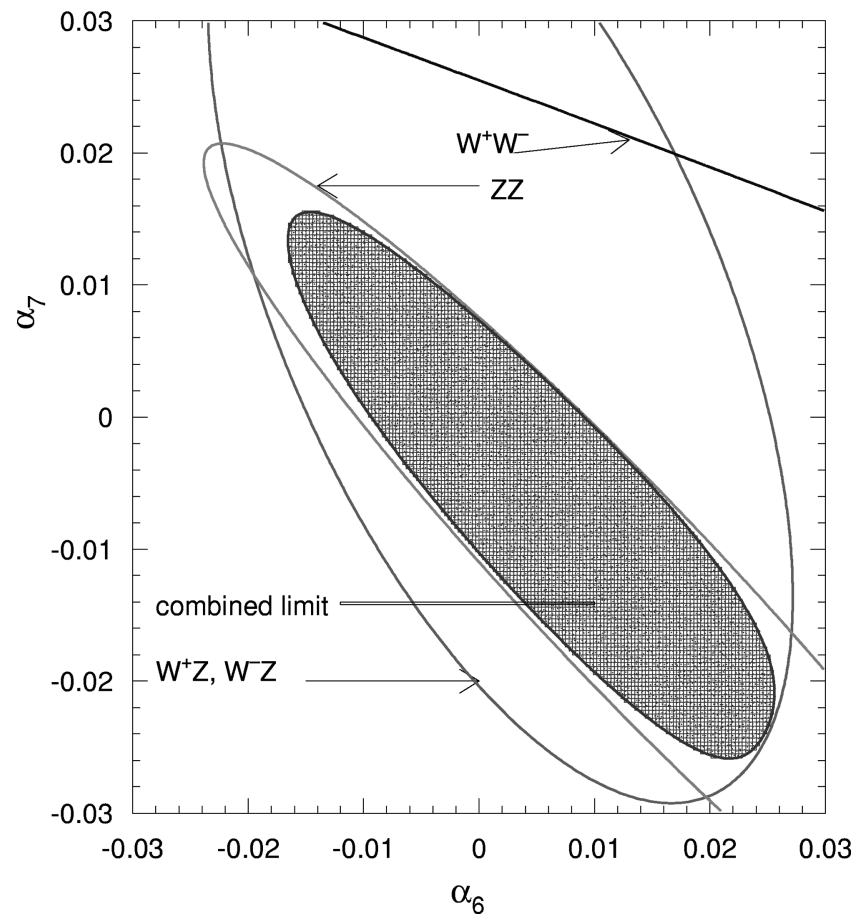

FIG. 5. 1- $\sigma$ C.L. exclusion region in the $\alpha_{6} \times \alpha_{7}$ plane from the $W^{+} W^{-}, W^{ \pm} Z$, and $Z Z$ productions. We applied all cuts and efficiencies discussed in the text and assumed that $\alpha_{4}=\alpha_{5}=\alpha_{10}=0$ and an integrated luminosity of $100 \mathrm{fb}^{-1}$.

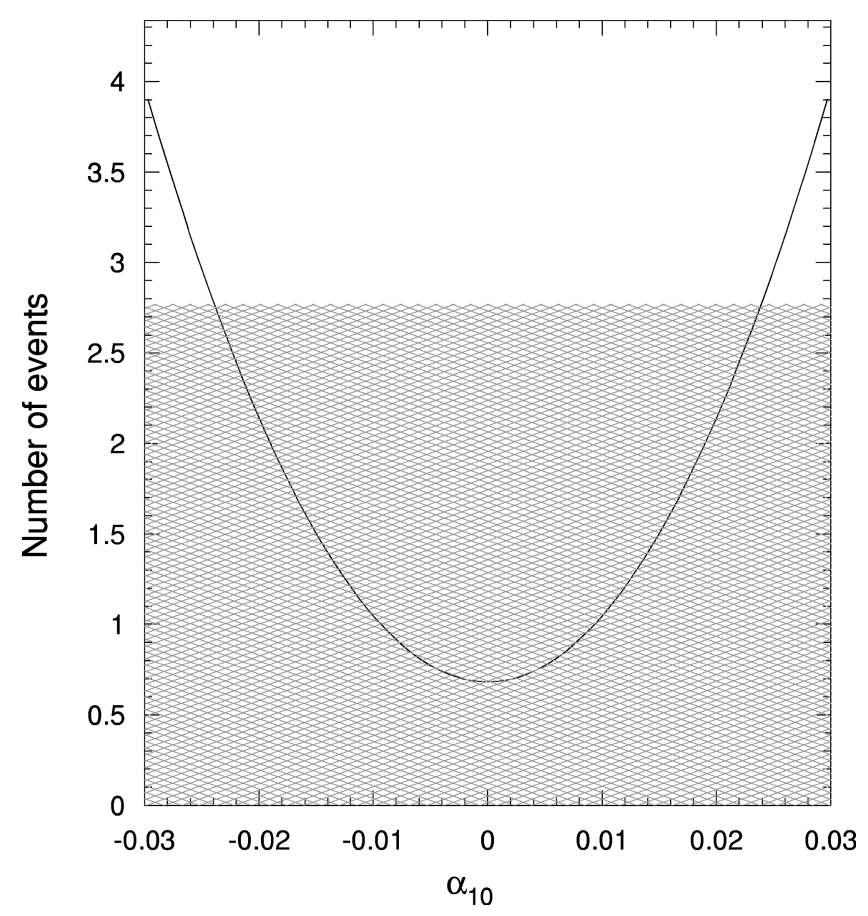

FIG. 6. Number of events for $Z Z$ production as a function of $\alpha_{10}$ where the horizontal line represents a $64 \%$ C.L. effect. We applied all cuts and efficiencies discussed in the text and assumed that $\alpha_{4}$ $=\alpha_{5}=\alpha_{6}=\alpha_{7}=0$ and an integrated luminosity of $100 \mathrm{fb}^{-1}$. 
TABLE III. 1- $\sigma$ limits on the anomalous quartic couplings $\alpha_{i}$ which will be accessible at LHC, as well as the present indirect bounds from Ref. [13]. The last column gives the luminosity required to get $90 \%$ C.L. limits inside the unitarity bounds $\left|\alpha_{i}\right|$ $\leqq 0.005$.

\begin{tabular}{lccc}
\hline \hline Coupling & $\begin{array}{c}\text { Indirect limits } \\
\left(\times 10^{-3}\right)\end{array}$ & $\begin{array}{c}\text { LHC limits } \\
\left(\times 10^{-3}\right)\end{array}$ & $\mathcal{L}\left(\mathrm{fb}^{-1}\right)$ \\
\hline$\alpha_{4}$ & $-120 \leqslant \alpha_{4} \leqslant 11$ & $-1.1 \leqslant \alpha_{4} \leqslant 11$ & 1750 \\
$\alpha_{5}$ & $-300 \leqslant \alpha_{5} \leqslant 28$ & $-2.2 \leqslant \alpha_{5} \leqslant 7.7$ & 750 \\
$\alpha_{6}$ & $-20 \leqslant \alpha_{6} \leqslant 1.8$ & $-9.6 \leqslant \alpha_{6} \leqslant 9.1$ & 2000 \\
$\alpha_{7}$ & $-19 \leqslant \alpha_{7} \leqslant 1.8$ & $-10 \leqslant \alpha_{7} \leqslant 7.4$ & 2000 \\
$\alpha_{10}$ & $-21 \leqslant \alpha_{10} \leqslant 1.9$ & $-24 \leqslant \alpha_{10} \leqslant 24$ & 2500 \\
\hline \hline
\end{tabular}

7, and 10, taking into account our results presented in Figs. 4, 5, and 6. These limits were obtained under the assumption that only one anomalous parameter is nonvanishing. For the sake of comparison, this table also contains the present indirect bounds on these anomalous couplings obtained from the precision measurements at the $Z$ pole [13] for a scale of new physics $\Lambda=2 \mathrm{TeV}$. As we can see, the direct bounds on $\alpha_{4}$ and $\alpha_{5}$ that can be obtained from the $V V j j$ production at LHC are more restrictive than the present limits by one order of magnitude in some cases. Nevertheless, the attainable direct limits on the $\mathrm{SU}(2)_{C}$ violating interactions $\alpha_{6}, \alpha_{7}$, and $\alpha_{10}$ are of the same order of the present indirect limits. Of course, some of the obtained limits are to be taken with a pinch of salt as they lay very close to the unitarity bounds for the anomalous couplings $\left(\left|\alpha_{i}\right| \lesssim 0.005\right)$. Therefore, we have also estimated the required luminosity to get $90 \%$ C.L. limits inside the unitarity bounds. This is presented in the last column of Table III.

It is also important to devise a strategy to disentangle the anomalous couplings in case a departure from the SM prediction is observed. In fact the simultaneous analysis of the $W^{ \pm} W^{ \pm} j j, W^{+} W^{-} j j, W^{ \pm} Z j j$, and $Z Z j j$ productions allows us to narrow down the anomalous couplings associated with the observed effect. The anomalous couplings $\alpha_{4}$ and $\alpha_{5}$ possess the distinctive characteristic of giving rise to observable effects for all processes $V V j j$. On the other hand, the couplings $\alpha_{6}$ and $\alpha_{7}$ lead to large signals in the channels $W^{ \pm} Z$ and $Z Z$ without any excess in the $W^{ \pm} W^{ \pm}$reaction. Finally the anomalous coupling $\alpha_{10}$ gives rise only to an excess of events in the $Z Z$ channel. The effects of $\alpha_{4}$ and $\alpha_{5} \quad\left(\alpha_{6}\right.$ and $\left.\alpha_{7}\right)$ can only be separated if we have additional information like the triple gauge-boson production at the NLC, where the $\alpha$ 's appears in different combinations for the different channels.

\section{CONCLUSIONS}

In this paper we presented the first complete calculation of the reaction $p p \rightarrow V V j j$ taking into account anomalous quartic vector-boson couplings. Our calculations were done at tree level in two different gauges and without any approximation, such as the effective $W$ one or the equivalence theorem. Our results show the ability of the LHC to shed some light on the electroweak symmetry-breaking sector and to look for a possible signal of strongly interacting electroweak symmetry breaking.

The attainable LHC limits for the quartic anomalous parameters are tighter than the present indirect bounds $[13,14]$, improving them by one order of magnitude in some cases. The LHC bounds are also one order of magnitude better than those which could be obtained from the study of triple gauge-boson production at the Next Linear Collider (NLC) $[6,9,10]$. Notwithstanding, the study of the reaction $V V \ell \ell(\ell=e$ or $\nu)$ at the NLC running at $\mathrm{TeV}$ energies $[8,9]$ will be able to improve the LHC limits by a factor of 2 to 8 , depending on the specific couplings.

The above results should be interpreted with care since the attainable bounds on the quartic couplings are very close to the unitarity limits for the anomalous couplings. In order to establish the real potentiality of the LHC to study these anomalous couplings, we have also estimated the required luminosity to get $90 \%$ C.L. limits inside the unitarity bounds, see Table III.

In our analyses, we assumed that the detection efficiencies of electrons and muons are the ones obtained from the production of heavy Higgs bosons. For a more realistic study one should construct a complete Monte Carlo generator including the vector-boson decays and detector resolution [27]. Such a generator will allow us not only to improve the leptonic cuts but also to study the hadronic decay channels of one of the gauge bosons, which could improve the limits on the anomalous couplings. We believe that even assuming this more realistic situation, the bounds presented in this paper will not change significantly.

\section{ACKNOWLEDGMENTS}

A.S.B. is grateful to A. Pukhov for important improvements of the COMPHEP package and to A. Solomin for the help in computing facilities. This work was supported by Fundação de Amparo à Pesquisa do Estado de São Paulo (FAPESP), by Conselho Nacional de Desenvolvimento Científico e Tecnológico (CNPq), by Programa de Apoio a Núcleos de Excelência (PRONEX), by DGICYT under Grant No. PB95-1077, and by CICYT under Grant No. AEN961718.
[1] See for instance, the LEP Collaborations ALEPH, DELPHI, L3, OPAL, the LEP Electroweak Working Group, and the SLD heavy flavor group, CERN-PPE/97-154, December 1997.

[2] B. Lee, C. Quigg, and H. Thacker, Phys. Rev. Lett. 38, 883
(1977); Phys. Rev. D 16, 1519 (1977); D. Dicus and V. Mathur, ibid. 7, 3111 (1973).

[3] J. Bagger, S. Dawson, and G. Valencia, Nucl. Phys. B399, 364 (1993). 
[4] J. Bagger et al., Phys. Rev. D 49, 1246 (1994); 52, 3878 (1995).

[5] A. Dobado and M. J. Herrero, Phys. Lett. B 228, 495 (1989); J. F. Donoghue and C. Ramirez, ibid. 234, 361 (1990); A. Dobado, D. Espriu, and M. J. Herrero, Z. Phys. C 50, 205 (1991); A. Dobado, M. J. Herrero, E. Ruiz, M. T. Urdiales, and J. R. Pelaez, Phys. Lett. B 352, 400 (1995); A. Dobado and M. T. Urdiales, Z. Phys. C 17, 965 (1996); J. R. Pelaez, Phys. Rev. D 55, 4193 (1997).

[6] S. Dawson, A. Likhoded, G. Valencia, and O. Yushchenko, BNL-HET-SD-7, in the Proceedings of 1996 DPF/DPB Summer Study on New Directions for High-Energy Physics, Snowmass, CO, hep-ph/9610299.

[7] V. Barger, K. Cheung, T. Han, and R. J. N. Philips, Phys. Rev. D 52, 3815 (1995).

[8] E. E. Boos, H. J. He, W. Kilian, A. Pukhov, and P. M. Zerwas, Phys. Rev. D 57, 1553 (1997).

[9] H. J. He, MSU-HEP-71120, hep-ph/9804210.

[10] G. Bélanger and F. Boudjema, Phys. Lett. B 288, 201 (1992); S. Dawson, et al., Proceedings of the 1996 DPF/DPB Summer Study on New Directions for High Energy Physics, Snowmass, CO, June 25-July 12, 1996, hep-ph/9610299; O. J. P. Éboli, M. C. Gonzalez-Garcia, and J. K. Mizukoshi, Phys. Rev. D 58, 034008 (1998); T. Han, H.-J. He, and C.-P. Yuan, Phys. Lett. B 422, 294 (1998).

[11] O. J. P. Éboli, M. C. Gonzalez-Garcia, and S. F. Novaes, Nucl. Phys. B411, 381 (1994).

[12] G. Bélanger and F. Boudjema, Phys. Lett. B 288, 210 (1992); O. J. P. Éboli, M. B. Magro, P. G. Mercadante, and S. F. Novaes, Phys. Rev. D 52, 15 (1995).

[13] P. Hernandez and J. Vegas, Phys. Lett. B 307, 116 (1993); S. Lietti, O. J. P. Éboli, M. C. Gonzalez-Garcia, and S. F. Novaes, ibid. 339, 119 (1994); A. Brunstein, O. J. P. Éboli, and M. C. Gonzalez-Garcia, ibid. 375, 233 (1996); S. Alam, S. Dawson, and R. Szalapski, Phys. Rev. D 57, 1577 (1998).
[14] Hong-Jian He, Yu-Ping Kuang, and C. P. Yuan, DESY-97056, Lectures given at CCAST Workshop on Physics at TeV Energy Scale, Beijing, China, 15-26 July 1996, hep-ph/9704276.

[15] J. M. Cornwall, D. N. Levin, and G. Tiktopoulos, Phys. Rev. D 10, 1145 (1974); C. E. Vayonakis, Lett. Nuovo Cimento 17, 383 (1976); B. W. Lee, C. Quigg, and H. B. Thacker, Phys. Rev. D 16, 1519 (1977); M. S. Chanowitz and M. K. Gaillard, Nucl. Phys. B261, 379 (1985).

[16] G. L. Kane, W. W. Repko, and W. B. Rolnick, Phys. Lett. 148B, 367 (1984); S. Dawson, Nucl. Phys. B249, 42 (1985).

[17] T. Appelquist and C. Bernard, Phys. Rev. D 22, 200 (1980); A. Longhitano, ibid. 22, 1166 (1980); Nucl. Phys. B188, 118 (1981).

[18] M. S. Chanowitz, M. Golden, and H. Georgi, Phys. Rev. D 36, 1490 (1987).

[19] H. Murayama, I. Watanabe, and K. Hagiwara, KEK report 91-11 (unpublished).

[20] W. Long and T. Steltzer, Comput. Phys. Commun. 81, 357 (1994).

[21] E. E. Boos, M. N. Dubinin, V. A. Ilyin, A. E. Pukhov, and V. I. Savrin, SNUTP-94-116, hep-ph/9503280; P. A. Baikov et al., hep-ph/9701412.

[22] V. Barger et al., Phys. Rev. D 42, 3052 (1990).

[23] U. Baur and E. W. N. Glover, Nucl. Phys. B347, 12 (1990); U. Baur and E. W. N. Glover, Phys. Lett. B 252, 683 (1990); V. Barger et al., Phys. Rev. D 42, 3052 (1990); V. Barger et al., ibid. 44, 1426 (1991); V. Barger et al., ibid. 46, 2028 (1992).

[24] ATLAS Technical Proposal, CERN/LHCC/94-43; ATLAS Internal Note, PHYS-NO-103.

[25] A. Abbasabadi, W. W. Repko, D. A. Dicus, and R. Vega, Phys. Rev. D 38, 2770 (1988); I. Kuss, ibid. 55, 7165 (1997).

[26] CTEQ Collaboration, MSUHEP-41024.

[27] A. S. Belyaev, O. J. P. Éboli, M. C. Gonzalez-Garcia, J. K. Mizukoshi, and S. F. Novaes (unpublished). 